\title{
LA MOVILIDAD GEOGRÁFICA DE LOS TRABAJADORES TRANSFRONTERIZOS DE EUROPA DEL ESTE EN LAS DOS ÚLTIMAS DÉCADAS*
}

\begin{abstract}
Resumen
La cifra de personas que trabajan en un país distinto al de residencia aumenta de forma extraordinaria en Europa y lo hace con mayor intensidad en los países del Este que, en la actualidad, proporcionan cerca de la mitad de los trabajadores transfronterizos. El artículo indaga en las tendencias recientes de la movilidad geográfica de este colectivo y en los factores que la impulsan. El texto se apoya en los trabajos de investigadores de diferentes países y en la información estadística que proporcionan Eurostat y la Comisión Europea. El aumento de los flujos se relaciona, entre otros factores, con la ampliación de la Unión Europea, la plena libertad de circulación y las diferencias socioeconómicas entre los países de origen y los de destino. La mayor parte de los desplazamientos cubren distancias cortas y son marcadamente asimétricos. En la movilidad intraeuropea, los países poscomunistas proporcionan mano de obra a los países occidentales. Alemania y Austria son los destinos más importantes debido, entre otros motivos, a su posición geográfica próxima a los países de origen.

Palabras clave: diferencias regionales; Europa del Este; flujos asimétricos; movilidad geográfica; trabajadores transfronterizos.
\end{abstract}

a Departament de Geografia. Universitat de València. Av. Blasco Ibáñez, 28. 46010 València. raviruel@uv.es

Fecha de recepción: 28/5/20. Fecha de aceptación: 27/7/20.

* El artículo desarrolla y actualiza parte de la comunicación "Migrantes en movimiento: trabajadores fronterizos y trabajadores desplazados en Europa", presentada por el autor en el IX Congreso de Migraciones, celebrado en la Universitat Autònoma de Barcelona, el 4, 5 y 6 de septiembre de 2019. 


\title{
THE GEOGRAPHICAL MOBILITY OF CROSS-BORDER WORKERS FROM EASTERN EUROPE IN THE LAST TWO DECADES
}

\begin{abstract}
The number of people working in a country other than the country of residence is increasing dramatically in Europe, and is doing so more sharply in Eastern countries, which currently provide nearly half of cross-border workers. This article examines the recent trends in this collective's geographic mobility and the factors that drive it. The text is based on the work of researchers from different countries and on the statistical information provided by Eurostat and the European Commission. The increase in flows is related, among other factors, to the enlargement of the European Union, full freedom of movement and socioeconomic differences between countries of origin and destination. Most of the trips cover short distances and are markedly asymmetrical. In intra-European mobility, post-communist countries provide labour to western countries. Germany and Austria are the most important destinations due, among other reasons, to the geographical position close to the countries of origin.

KeYwords: asymmetric flows; cross-border workers; Eastern Europe; geographic mobility; regional differences.
\end{abstract}

\section{INTRODUCCIÓN}

La emigración, trabajar y residir en un país distinto al de origen, es la principal modalidad de movilidad laboral en Europa. En la actualidad, cerca de 10 millones de ciudadanos de la Unión Europea (UE) trabajan o buscan empleo en un país que no es el suyo, lo que supone el $4 \%$ de la población ocupada (Friers-Tersch, Jones, Böök, de Keyser, \& Tugran, 2020). A estos hay que añadir los trabajadores transfronterizos, que viven en un país y trabajan en otro (European Commission, 2011). El emigrante reubica el lugar de trabajo y el de residencia en otro país, y lo hace pocas veces a lo largo de la vida activa. El trabajador transfronterizo se mueve a través de las fronteras yendo con relativa frecuencia entre el país de residencia y el de trabajo.

El análisis de la movilidad transfronteriza es complejo, ya que hay una gran variedad de desplazamientos (diarios, semanales, de unos meses, temporales, circulares, etc.) y muchos de ellos son opacos al registro estadístico. Disponemos de información interesante de los trabajadores fronterizos, que cruzan la frontera a diario o, al menos, una vez a la semana, y de los trabajadores que son desplazados por sus empresas para realizar una actividad en otro país por tiempo determinado. Los primeros se mueven en virtud de la libre circulación de trabajadores. Los desplazados lo hacen en el marco de la libre circulación de servicios. En la actualidad, hay dos millones de trabajadores fronterizos en Europa y los desplazados realizan cerca de tres millones de desplazamientos. Las cifras son modestas, entre unos y otros no llegan a representar el 2\% de la población ocupada (De Wispelaere, De Smedt, \& Pacolet, 2020; Friers-Tersch et al., 2020). La movilidad transfronteriza aumenta de forma extraordinaria en las dos últimas décadas en relación con la ampliación de la UE hacia el Este, la libre circulación de trabajadores y servicios y las grandes diferencias socioeconómicas entre los países de residencia y los de 
trabajo. Los flujos convergen en Europa occidental, donde se concentra la oferta de empleo. Los países poscomunistas participan como proveedores de mano de obra.

El objetivo de este artículo es analizar la movilidad geográfica de los trabajadores transfronterizos de Europa del Este ${ }^{1}$ y los factores que han contribuido al extraordinario aumento de los desplazamientos en fecha reciente. El texto presenta, en primer lugar, el marco conceptual y las fuentes de información utilizadas. A continuación, se hace una breve retrospectiva de la movilidad transfronteriza. Después, se ocupa del rápido aumento de los flujos y de los factores que coadyuvan al notable aumento de la movilidad Este-Oeste. El artículo finaliza con unas reflexiones sobre la relevancia del trabajo transfronterizo.

\section{LA MOVILIDAD: TRABAJADORES FRONTERIZOS Y TRABAJADORES DESPLAZADOS}

Una gran parte de las migraciones actuales son de carácter temporal y no se pueden identificar con la definición clásica de emigración, entendida como ruptura o cambio de residencia definitivo o de larga duración (al menos, de un año). Además de la emigración, hay desplazamientos que implican frecuentes idas y venidas, breves y repetidos periodos de permanencia en el extranjero. El término movilidad expresa mejor el carácter "líquido" o "fluido" de los desplazamientos pendulares, de corta duración, temporales, circulares, intermitentes o cíclicos (Engbersen, Leerkes, Grabowska-Lusinska, Snel, \& Burgers, 2013; Mintchev, Boshnakov, Richter, \& Ruspini, 2016).

Las estadísticas de migraciones no proporcionan información de la duración de los desplazamientos, no distinguen entre la migración a corto plazo y la migración a largo plazo, por lo que analizar la movilidad de los trabajadores transfronterizos es una tarea compleja. El análisis de modalidades específicas, como el trabajador fronterizo y el trabajador desplazado, figuras tipificadas en las normas comunitarias, proporciona una aproximación a la movilidad transfronteriza en Europa. Para ello, lo más aconsejable es recurrir a los datos que proporcionan Eurostat y la Comisión Europea y a los resultados de investigaciones empíricas que han llevado a cabo autores de diferentes regiones y países. Interesa señalar que algunas de las conclusiones a las que llegan no se pueden comparar, ya que no se refieren exactamente a lo mismo cuando estudian la migración temporal, la circular o el retorno, modalidades de migración que tampoco están claramente definidas en la mayor parte de los Estados europeos (Fries-Tersch et al., 2020).

Los informes publicados por la Dirección General de Empleo, Asuntos Sociales e Inclusión de la Comisión dedican especial atención a los trabajadores transfronterizos (Fries-Tersch et al., 2020). La movilidad de los trabajadores desplazados se estudia aparte (De Wispelaere et al., 2020). Los trabajadores transfronterizos (cross-border workers) viven en un país y trabajan en otro, cruzan las fronteras con regularidad. La definición no hace referencia a la distancia recorrida por el trabajador y tampoco al carácter limítrofe o no de los territorios implicados, pero la mayor parte son trabajadores fronterizos

1 La expresión "Europa del Este" se refiere a los países poscomunistas (11 en total) que se han incorporado a la Unión Europea en el siglo XXI. 
(frontier worker) stricto sensu. El Reglamento CE/883/2004² (artículo 1f, p. 12) define el trabajador fronterizo como "toda persona que realice una actividad por cuenta ajena o propia en un Estado miembro y resida en otro Estado miembro al que regrese normalmente a diario o al menos una vez por semana". Algunos investigadores proponen ampliar el concepto a los desplazamientos semanales, mensuales o estacionales (Nerb, Hitzelsberger, Woidich, Pommer, Hemmer, \& Heczko, 2009; Verwiebe, Reinprecht, Haindorfer, \& Wiesboeck, 2017). Según el último informe de la OCDE (2019) sobre las migraciones internacionales, el trabajo desplazado es la principal modalidad de migración laboral temporal en Europa. La Directiva 96/71/CE³ (artículo 2.1, p. 3), define al trabajador desplazado (posting of workers) como la persona que "durante un periodo limitado realice un trabajo en el territorio de un Estado miembro distinto de aquel en cuyo territorio trabaje habitualmente". Parte de este colectivo trabaja en otro Estado (Reglamento CE/883/2004, artículo 12, p. 24) y parte lo hace en dos o más Estados (artículo 13, p. 25 )4.

Las idas y venidas cotidianas de los trabajadores fronterizos son más probables cuando el lugar de trabajo y el de residencia están cerca de la frontera, es decir, cuando la distancia a recorrer es relativamente corta, como ocurre entre Eslovaquia y Hungría, entre regiones situadas a uno y otro lado del Danubio (Balogh \& Pete, 2018). Sin embargo, algunos trabajadores cubren distancias de centenares o miles de kilómetros, por ejemplo, de Polonia a los Países Bajos o de Rumania a Italia o España. En este caso, disminuye la frecuencia de los desplazamientos, siendo más habitual que se produzcan una vez a la semana. El desplazamiento, cuya duración media es de tres meses, es compatible con trayectos que cubren distancias más largas. Dado que una misma persona puede realizar varias salidas al exterior, constituye un ejemplo de migración circular, que se define como la repetición de migraciones por la misma persona entre dos o más países ${ }^{5}$.

El trabajador fronterizo cruza las fronteras en virtud de la libre circulación de trabajadores y se somete a la legislación laboral del país de destino. En cambio, el trabajador desplazado se mueve en el marco de la libre circulación de servicios y sigue estando afiliado a la Seguridad Social del país de origen, donde tiene su residencia principal y donde trabaja de forma habitual. La empresa le garantiza el salario mínimo del país de acogida, pero no disfruta de los mismos derechos laborales y beneficios que los trabajadores locales. Los desplazados son trabajadores más flexibles, a los que se recurre con frecuencia para aumentar la plantilla cuando lo requiere la producción. Esta modalidad de movilidad ha sido muy criticada porque, en general, las condiciones laborales son muy precarias y porque contri-

2 Reglamento (CE) no 883/2004 del Parlamento Europeo y del Consejo, de 29 de abril de 2004, sobre la coordinación de los sistemas de seguridad social. Diario Oficial de la Unión Europea, de 30 de abril de 2004. Recuperado a partir de https://www.boe.es/doue/2004/166/L00001-00123.pdf

3 Directiva 96/71/CE del Parlamento Europeo y del Consejo, de 16 de diciembre de 1996, sobre el desplazamiento de trabajadores efectuado en el marco de una prestación de servicios. Diario Oficial de las Comunidades Europeas, $\mathrm{n}^{\circ} \mathrm{L}$ 18, de 21 de enero de 1997. Recuperado a partir de https://eur-lex.europa.eu/legal-content/ES/TXT/PDF/?uri $=$ CELEX:31996L0071\&from=ES

4 Hay otras modalidades de trabajadores desplazados, entre las que destacan los funcionarios, con pocos efectivos. En 2018, la categoría "otros", registró algo más de 55.000 solicitudes, el 1,8\% del total.

5 Véase el Glosario sobre Migración y Asilo de la Red Europea de Migraciones (EMN, 2012). Recuperado a partir de https://ec.europa.eu/home-affairs/what-we-do/networks/european_migration_network/glossary_en 
buye a la consolidación de los mercados de trabajo duales en los países de acogida (Cremers, Dølvik, \& Bosch, 2007). El trabajador fronterizo es, por el contrario, menos vulnerable al dumping social y al fraude, ya que comparte derechos laborales con los trabajadores autóctonos y como ellos está afiliado a la Seguridad Social del país de acogida.

La Comisión Europea aporta información de los países miembros de la UE y de la EFTA ${ }^{6}$. Los datos de trabajadores fronterizos se refieren a personas, mientras que los relativos a trabajadores desplazados registran movimientos y hay que tener en cuenta que un mismo trabajador puede desplazarse varias veces al año. Algunos autores consideran que el trabajo desplazado está subestimado porque no todos los que se desplazan lo comunican (Lends, Mussche, \& Marx, 2019; Mussche, Corluy, \& Marx, 2016; Riesco, García, \& Maira, 2018). La información se ofrece por país de origen y país de destino, salvo en el caso de los desplazados que trabajan en dos o más Estados (Reglamento CE/883/2004, artículo 13), en el que solo consta el país de salida. No todos los países aportan información de los sectores de actividad y de la duración de la estancia de trabajo. La propia Comisión reconoce que la información disponible no es exhaustiva, pero permite analizar las tendencias y las diferencias entre países (De Wispelaere et al., 2020). Los datos permiten cartografiar los trayectos que realizan los trabajadores transfronterizos dentro del espacio UE-EFTA. Para la elaboración de las figuras se han utilizado herramientas de diseño gráfico digital estándar.

La información hace referencia al país de procedencia del trabajador, no al de nacimiento y/o nacionalidad. Entre los fronterizos, hay trabajadores que trasladan su residencia al país vecino, donde las condiciones de vida son más atractivas, y acuden a trabajar al país de procedencia. Algunos inmigrantes, tras perder el empleo en el país de residencia, han encontrado una nueva oportunidad al otro lado de la frontera. También hay ciudadanos de países terceros que acceden a la zona UE-EFTA como trabajadores desplazados porque de esta forma eluden las restricciones a la inmigración, es decir, les resulta más fácil moverse en virtud de la libre circulación de servicios que en el marco de la libre circulación de trabajadores (Cremers, 2014; Lends et al., 2019; Mussche et al., 2016).

La investigación aumenta a medida que lo hace la movilidad transfronteriza. El fenómeno se ha estudiado fundamentalmente en regiones de Europa occidental, donde tiene mayor importancia social y económica, y en fecha reciente en países de Europa central y oriental, donde el fenómeno se incrementa de forma rápida (Balogh \& Pete, 2018; Baumgartner \& Forster, 2017; Sert, 2018; Verwiebe et al., 2017). Las investigaciones indagan en los factores que contribuyen a estos desplazamientos y en las condiciones de vida y de trabajo, entre otros aspectos. La migración temporal de los trabajadores

6 Los últimos datos disponibles corresponden a 2018, cuando el Reino Unido seguía formando parte de la UE, de la que salió el 31 de enero de 2020. Por ello, el marco espacial de referencia es el integrado por la UE-28. Al núcleo original (la Comunidad Económica Europea de 1957), formado por Alemania, Bélgica, Luxemburgo, Francia, Italia y los Países Bajos, se han ido sumando, sucesivamente, el Reino Unido, Irlanda y Dinamarca (1973), Grecia (1981), España y Portugal (1986), Austria, Finlandia y Suecia (1995), Estonia, Letonia, Lituania, Eslovenia, Chequia, Eslovaquia, Hungría, Polonia, Chipre y Malta (2004), Bulgaria y Rumania (2007) y Croacia (2013). Por su parte, Noruega, Islandia, Suiza y Liechtenstein componen la Asociación Europea de Libre Comercio (EFTA). Los 32 países (UE+EFTA) conforman un espacio de libre circulación de trabajadores. 
desplazados se relaciona con la creciente desregulación del mercado del trabajo, la transformación estructural del empleo, la flexibilidad, la externalización/subcontratación y la informalización, y con la precariedad de las migraciones (Danaj, 2018; Perocco, 2018).

\section{UN FENÓMENO DE LARGA TRADICIÓN}

La movilidad transfronteriza está profundamente enraizada en la historia económica del continente europeo. En otras épocas, las ausencias del lugar de residencia se prolongaban varios meses debido no tanto a las distancias recorridas como a los medios de transporte utilizados. En Europa central hay una larga tradición de flujos fronterizos desde la monarquía de los Habsburgo (Wiesböck, 2016). La emigración temporal de trabajadores adquirió relevancia en los siglos XVIII y XIX, cuando miles de campesinos se dirigían desde las áreas rurales de baja productividad agraria y oferta de empleo insuficiente a las regiones agrícolas de monocultivo, elevada productividad y salarios atractivos (Bade, 2003). Así, por ejemplo, la Alemania de finales del ochocientos recibía a "los trabajadores de temporada polacos, riadas nómadas reclutadas en Rusia por algunos agentes, que pasan la frontera a millares en primavera para volver a marchar en otoño" (Weber, 1991: 76). Los temporeros proporcionaban la mano de obra necesaria en época de recolección. Los empleadores preferían a los inmigrantes estacionales por ser una mano de obra barata y flexible, con la que conseguían un sustancial ahorro en vivienda y en cargas sociales.

En los primeros años del siglo XX los emigrantes del Este se dirigían a países americanos y en el periodo de entreguerras predominaron los flujos intraeuropeos. La emigración ni siquiera se interrumpió durante el largo periodo de la Guerra Fría, cuando los regímenes comunistas se esforzaron por evitar la salida de sus ciudadanos al considerar la emigración como una deserción. Los polacos, por ejemplo, no podían tener el pasaporte en casa y antes de obtenerlo se sometían a una minuciosa investigación por parte de los servicios encargados del Estado (Dac, 2015). Pese al férreo control de las autoridades, millones de personas lograron emigrar a Occidente, muchos de ellos como disidentes políticos, convencidos de que jamás regresarían o no lo harían en mucho tiempo. A esta emigración definitiva habría que añadir los desplazamientos temporales entre países del bloque del Este, que se contemplaban como la transferencia de mano de obra para el uso coordinado de recursos entre países amigos (Viruela, 2004).

Tras la caída del muro de Berlín (noviembre de 1989), los ciudadanos del Este recuperaron la libertad de viajar al extranjero. Desde entonces, los flujos Este-Oeste se perfilan fundamentalmente como migraciones de corta duración, ya que los migrantes pueden salir y volver a casa cuando lo deseen (Arango, 2003; Rea, 2013). La exención de visado tuvo un papel destacado en el aumento de estos desplazamientos. En 1991 se eximió de este requisito a polacos, húngaros, checos y eslovacos, a los que años después se añadieron los nacionales de otros países. Los migrantes podían entrar en Europa occidental, pero sin la libertad de residencia y trabajo. Muchos de ellos han aprovechado esta circunstancia para desplazarse legalmente como turistas, convirtiéndose en inmigrantes indocumentados cuando trabajan (en la economía sumergida) y/o prolongan su estancia durante más tiempo del permitido (tres meses), para lo que han contado con el apoyo logístico de las redes sociales integradas por familiares, amigos o grupos de origen común. Hace veinte años, el trabajo irregular de los "falsos turistas" fue la 
principal modalidad de migración temporal laboral para los emigrantes de Europa del Este (Salt, 2002). Polacos, húngaros, checos y eslovacos se desplazaban a Alemania y Austria, los búlgaros a Grecia, los croatas y eslovenos a Italia, etc. (Okolski, 2001a; Stacher, 2001). Miles de trabajadores realizaban continuas idas y venidas entre el lugar de origen y el de destino, trabajando temporalmente en la construcción, la agricultura o el servicio doméstico y asistencial. Era bastante habitual que familiares y amigos se alternasen en el mismo puesto de trabajo cada tres meses, como hacían las mujeres que se desplazaban a Alemania y Bélgica (Morokvasic, 1999; Rea, 2013).

Gran parte de los desplazamientos estaban protagonizados por los comerciantes de "maleta" (Wihtol, 2013). Se trata del pequeño comercio de ida y vuelta que ya se practicaba durante la Guerra Fría. Los migrantes aprovechaban la salida para comprar y vender una gran variedad de productos manufacturados, lo que les aportaba un pequeño beneficio por la diferencia de precios. El fenómeno estuvo bastante generalizado: polacos en Alemania; rusos, bielorrusos y ucranianos en Polonia; búlgaros en Rumania o Turquía; húngaros en Serbia, checos en Viena, etc. (De Tinguy, 1993; Williams \& Balá, 2002; Soultanova, 2005; Bioteau \& Michalon, 2008).

Aunque la mayor parte de los emigrantes trabajaba en la economía sumergida, miles de ellos lo hacían de forma legal gracias a los acuerdos bilaterales firmados con varios países: Alemania, Francia, Bélgica, Luxemburgo o España (SOPEMI, 1998, Pajares, 2007; Dac, 2015). El salario, superior al que podían obtener en el país de origen, compensaba la descualificación o movilidad laboral descendente que sufría el trabajador. Así, por ejemplo, un cirujano polaco, recogiendo bayas o plantando espárragos en Alemania, podía ganar un salario considerablemente más elevado que ejerciendo su profesión en Polonia (Bade, 2003).

A las migraciones temporales de varios meses, se añadían los desplazamientos de menor duración, de unas semanas o días, cuya frecuencia estaba muy condicionada por la proximidad geográfica. Algunos autores calificaron estos flujos de incompletos o casi migratorios, otros preferían el término más genérico de movilidad (Stola, 1998; Okólski, 2001b; Williams \& Bála, 2002). Los mismos protagonistas hablaban de viajes (Morockvasic, 1999). Aunque cada salida podía hacerse en pocos días, algunos migrantes pasaban la mayor parte del año en el extranjero (Salt \& Clark, 2001).

\section{Tendencias Recientes de la movilidad transfronteriza Este-Oeste}

En 2018 había en Europa más de 2 millones de trabajadores fronterizos, según Eurostat ${ }^{7}$, y los trabajadores desplazados realizaron cerca de 3 millones de movimientos (Friers-Tersch et al., 2020). Estas cifras duplican y triplican, respectivamente, las registradas unos años atrás. Los desplazamientos transfronterizos aumentan en toda Europa y lo hacen con mayor intensidad en los países del Este, de donde salen 4 de cada 10 trabajadores móviles (figura 1). La propensión a la movilidad de los ciudadanos de países poscomunistas atañe fundamentalmente al grupo de trabajadores fronterizos y a los desplazados que

7 Eurostat: Employment and commuting by NUTS 2 regions. Recuperado a partir de http://appsso.eurostat.ec.europa.eu/nui/show.do?dataset=lfst_r_lfe2ecomm\&lang=en 
trabajan en dos o más Estados (Reglamento CE/883/2004, artículo 13), que en dos años han duplicado el número de salidas. El aumento de este colectivo compensa la reducción de los que trabajan en otro Estado (artículo 12), categoría que moviliza a un mayor número de trabajadores de Europa occidental.

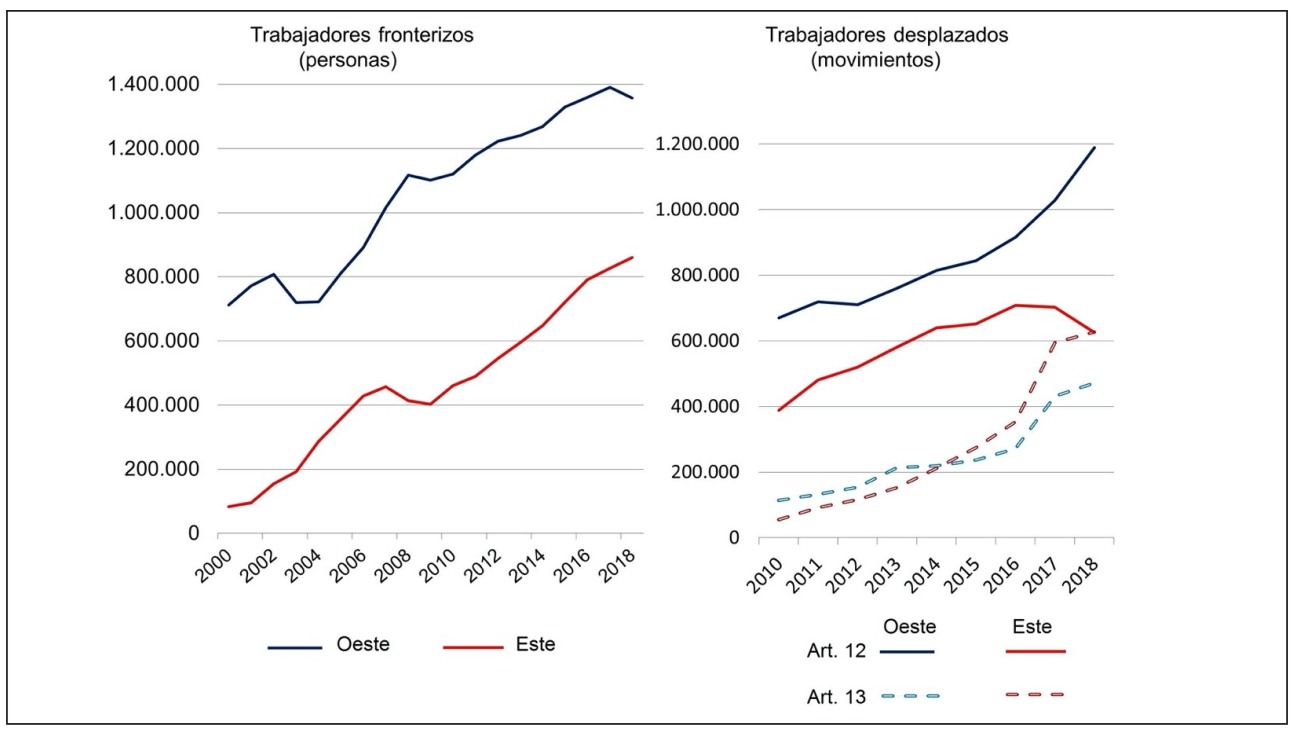

Figura 1. Evolución reciente de la movilidad transfronteriza por región de salida. Fuente: Eurostat: Employment and commuting by NUTS 2 regions, para los trabajadores fronterizos, y De Wispelaere et al., 2020, pp. 27 y 39. Elaboración propia.

El aumento de la movilidad ha ido acompañado de la ampliación del campo migratorio. En los mapas de las figuras 2 y 3 se representan, respectivamente, los flujos que suman o superan los 5.000 trabajadores fronterizos y los 5.000 movimientos de los desplazados. Se compara la situación del primer año del que hay datos (2006-07 y 2010, según el caso) con la información más reciente (2018), con el fin de apreciar los cambios que se han producido. Las cifras disponibles no permiten analizar los trayectos (país emisor-país receptor) de los trabajadores desplazados según el artículo 13 del Reglamento CE/883/2004 porque de este grupo no se proporciona información del país de destino.

Lo primero que constatamos es que, en la actualidad, el espacio de circulación de los dos grupos es similar y que en un corto periodo de tiempo quienes más lo han ensanchado han sido los trabajadores fronterizos. En efecto, hace quince años, en un contexto de escasa movilidad, sobresalían los flujos de Estonia a Finlandia y los que recibía Austria de Chequia, Eslovaquia y Hungría. En aquellos momentos, Alemania tenía muy poco atractivo, lo que se explica por la pobreza económica de la ex RDA, cuyo nivel de desempleo a principios del siglo XXI era considerablemente superior a la media estatal (Nerb et al., 2009). Sin embargo, recibía trabajadores desplazados, sobre todo desde Polonia, que también trasladaba operarios a otros países: Francia, Bélgica o los Países Bajos. 


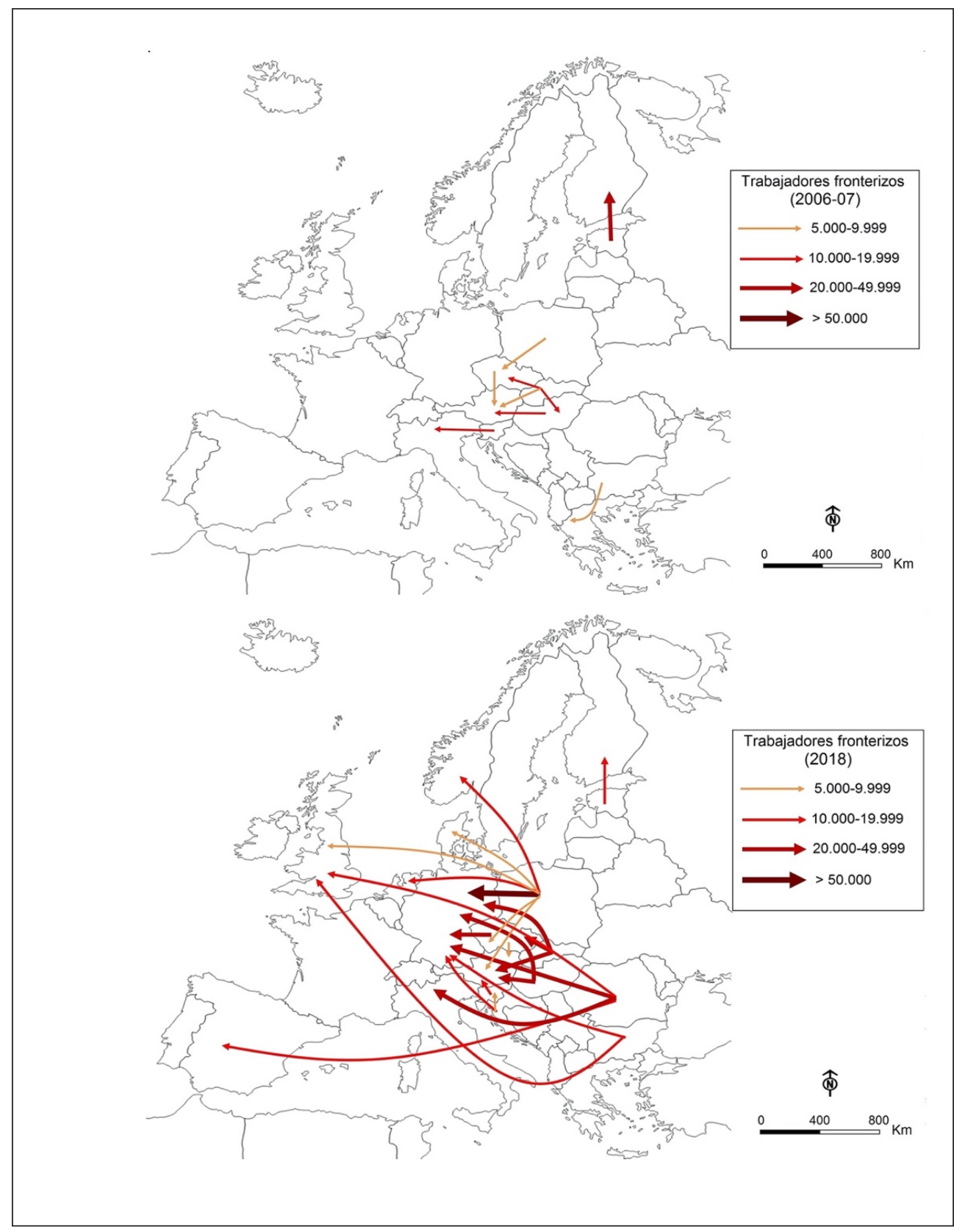

Figura 2. Principales flujos de trabajadores fronterizos con origen y destino en Europa del Este, en 2006/07 y 2018. Fuente: European Commission, 2008, p. 15; Friers-Tersch et al., 2020, pp.143144. Elaboración propia. 


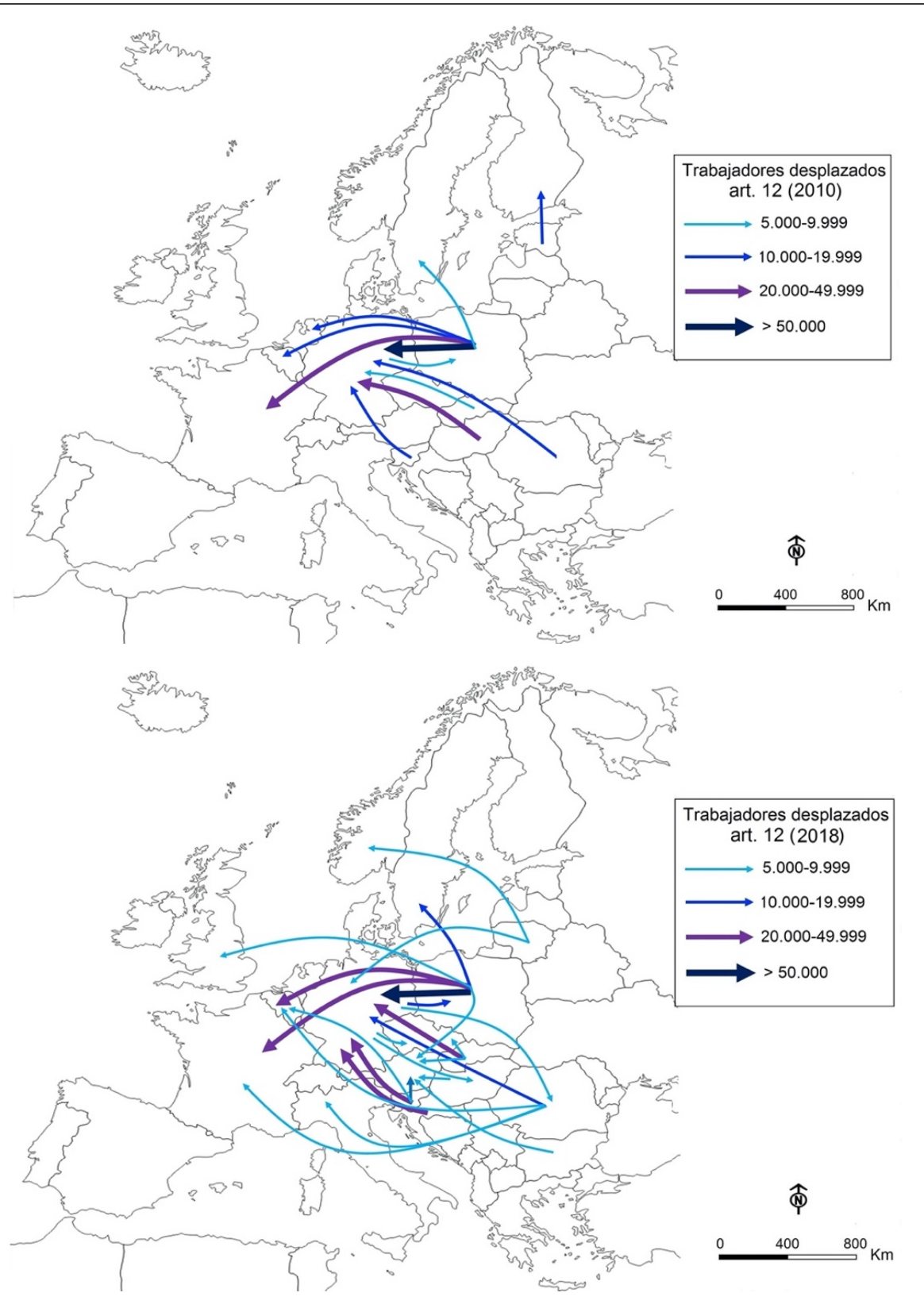

Figura 3. Principales flujos de trabajadores desplazados (artículo 12) con origen y destino en Europa del Este, en 2010 y 2018. Fuente: European Commission, 2012, p. 26; De Wiwpelaere et al., 2020, p. 24. Elaboración propia. 
En la primera década del siglo XXI, la emigración con carácter definitivo o de larga duración resultaba más atractiva que la movilidad transfronteriza, como revela el gran número de polacos que emigraron al Reino Unido o el extraordinario volumen de rumanos acogidos por Italia y España (Forti, Pittau, \& Ricci, 2004; Okólski \& Salt, 2014; Pajares, 2007). La movilidad transfronteriza también aumentó, pero lo hizo más en virtud de la libre circulación de servicios (desplazados) que en aplicación del derecho de libre circulación de trabajadores (fronterizos). Los flujos de trabajadores móviles han aumentado con las ampliaciones de la UE en 2004 y 2007 y se han dirigido fundamentalmente a Alemania y Austria (De Wiwpelaere et al., 2020; Verwiebe et al., 2017).

Alemania es, con diferencia, el principal mercado para los trabajadores fronterizos y desplazados que salen de Europa del Este, ya que acoge al 38\% de los fronterizos y al 50\% de los desplazados, con mayor representación de polacos. Austria también es un destino importante, en particular para los trabajadores fronterizos que salen de Hungría y Eslovaquia. En general, predominan los trayectos cortos. No obstante, algunos abarcan distancias más largas, como hacen los trabajadores fronterizos rumanos que acuden a Italia y España, y los búlgaros que llegan hasta el Reino Unido, así como los polacos que se desplazan a los Países Bajos, Bélgica o Francia en virtud de la libre circulación de servicios. A diferencia de los trabajadores de Europa occidental, los del Este viajan en mayor proporción a países que no limitan con el de origen (European Commission, 2011). Los mapas de las figuras 2 y 3 muestran el carácter asimétrico de los desplazamientos, ya que apenas hay flujos de dirección Oeste-Este. En consecuencia, los países poscomunistas actúan como exportadores de mano de obra, mientras que la oferta de empleo se concentra en Occidente.

El intercambio de trabajadores entre los países poscomunistas tiene poca importancia, lo que se explica por la debilidad estructural de las regiones fronterizas en países de economía muy centralizada en el pasado reciente y por las escasas diferencias de salario entre ellos (Nerb et al., 2009). Los únicos flujos de cierta consideración son los que relacionan Eslovaquia con Hungría y los que reciben la República Checa y Hungría (Balogh \& Pete, 2018; Ceccagno \& Sacchetto, 2020; Fries-Tersch et al., 2020).

Los trabajadores transfronterizos representan una fracción ínfima de la población ocupada, apenas el 2\% del empleo total. No obstante, la mano de obra móvil tiene gran importancia a escala regional y sectorial. Por una parte, es fundamental en muchos países y regiones de salida, en particular en Estonia, Hungría, Chequia y Eslovaquia, donde supone entre el 2 y el 5\% de los ocupados. En Eslovaquia hay casi tantos trabajadores transfronterizos como emigrados. Por otra parte, los europeos del Este constituyen el principal componente de la mano de obra móvil en Alemania, donde equivalen al $70 \%$ de todos los trabajadores transfronterizos, y en Austria, donde el 80\% de los trabajadores fronterizos procede de Hungría, Eslovaquia y otros países vecinos. Otros países occidentales acogen principalmente trabajadores de la UE-15 (Fries-Tersch et al., 2020; De Wispelaere et al., 2020).

Los trabajadores de Europa del Este se ocupan en diversos sectores de actividad. Los búlgaros y rumanos destacan en la industria cárnica y en la construcción de Alemania (Wagner, 2015). Los estonios trabajan en la construcción naval y en otras actividades en Finlandia (Danaj \& Sippola, 2015). Los húngaros y eslovacos en la hostelería o el servicio doméstico austriacos (Drbohlav \& Pavelková, 2018; 
Wiesböck, 2016). También tienen una destacada presencia en el transporte internacional de mercancías por carretera, la mayor parte de los conductores proceden de Polonia ${ }^{8}$ y trabajan en Alemania, Holanda, Francia, etc. (EFT, 2012; Riesco et al., 2018). En los últimos años, se ha incrementado la movilidad de profesionales de la sanidad: médicos rumanos cubren las guardias en centros hospitalarios de Bélgica, los estonios trabajan durante unos días a la semana en Finlandia, Noruega o Suecia, el Reino Unido recibe médicos y enfermeros de varios países del Este (Maier, Glinos, Wismar, Bremner, Dussault, \& Figueras, 2011).

La nacionalidad del trabajador transfronterizo no tiene porqué ser la del país de procedencia. La circulación de ciudadanos de países terceros por los mercados de trabajo del espacio UE-EFTA es un fenómeno en aumento. Los nuevos socios de la UE actúan como plataformas de distribución de trabajadores inmigrantes, más sumisos y baratos. Ciudadanos ucranianos trabajan en el sector de la construcción de Chequia por mediación de agencias polacas (Trčka, 2018). Eslovenia traslada a Alemania trabajadores de Macedonia, Serbia, Bosnia y Croacia9 (Bogoevski, 2016). Inmigrantes filipinos han llegado a Europa a través de Letonia para trabajar en el transporte de mercancías (Cremers, 2014). Esto es así porque, como ya se ha comentado, les resulta más fácil acceder a Europa como trabajadores desplazados que como inmigrantes.

\section{FACTORES QUE FAVORECEN LA MOVILIDAD TRANSFRONTERIZA}

Las ampliaciones de la UE, con la incorporación de ocho países poscomunistas en 2004, dos más en 2007 y el último en 2013, y la consiguiente apertura de fronteras han impulsado la movilidad transfronteriza Este-Oeste (Favell, 2008; Morokvasic, 2015; Skeldon, 2012; Verwiebe et al., 2017). Cuando los países poscomunistas se incorporaron a la UE, los antiguos socios, salvo unas pocas excepciones, aplicaron moratorias a la libre circulación de trabajadores porque temían una avalancha de inmigrantes procedentes de países con salarios y niveles de renta mucho más bajos (Kahanec, Pytliková, \& Zimmermann, 2016; Koikkalainen, 2011). Las restricciones limitaron los desplazamientos de miles de migrantes que, como reconocía la Comisión Europea (2008), se vieron empujados a la inmovilidad geográfica y al trabajo precario en la economía sumergida del país de destino. No obstante, los acuerdos bilaterales firmados con Alemania, Francia, Bélgica, Luxemburgo o España permitieron que miles de europeos del Este trabajaran temporalmente de forma legal en sectores como la agricultura, la construcción o la hostelería. Por otra parte, al no adoptarse medidas transitorias contra la libre circulación de

8 Véase Eurostat: Estatistics Explained. Transport Road. Recuperado a partir de https://ec.europa.eu/eurostat/statistics-explained/index.php?title=Category:Road

9 Eslovenia es el principal exportador de trabajadores de los Balcanes a Occidente. Véase Radio Slovenia (2017). Slovenia is the biggest exporter of foreign workers to the West. Recuperado a partir de https://val202.rtvslo. si/2017/05/slovenia-is-the-biggest-exporter-of-foreign-workers-to-the-west/ 
servicios y los trabajadores por cuenta propia ${ }^{10}$, los nuevos ciudadanos comunitarios podían desplazarse como trabajadores autónomos y como trabajadores desplazados desde el mismo día de la adhesión de sus países a la UE (Comte, 2019; Dølvik \& Eldring, 2006). Es decir, mientras los Estados occidentales imponían moratorias a la libre circulación de trabajadores, las empresas los reclutaban al amparo de la Directiva 96/71/CE.

El hecho de que el empleador no esté obligado a pagar al trabajador desplazado más que el salario mínimo del país de acogida y que las cotizaciones a la Seguridad Social se satisfagan en el país de origen supone una sustancial reducción de costes (Fernandes, 2017). La contratación de trabajadores desplazados de Europa del Este aumenta durante la crisis de 2008, salvo el breve paréntesis de los primeros años. La mano de obra barata, dispuesta a aceptar las condiciones laborales que rechazan los trabajadores autóctonos, permite a los empleadores reducir costes y flexibilizar las plantillas en función de la demanda. A las empresas del Reino Unido, España o Alemania, les resulta más barato contratar un fontanero polaco, un jornalero rumano o un carnicero búlgaro que un trabajador local (Cremers et al., 2007; Wagner, 2015). La reducción de costes es mayor cuando se trata de trabajadores de países terceros. Como ya se ha comentado, en Europa aumenta la presencia de inmigrantes que llegan como trabajadores desplazados a través de los nuevos socios comunitarios. Por ello, no es sorprendente que las salidas de trabajadores desplazados desde países del Este pasaran de menos de 500.000 en 2010 a 1,3 millones en la actualidad.

La permeabilidad de las fronteras ha contribuido a la movilidad geográfica. El notable aumento de los trabajadores fronterizos se produce al finalizar las restricciones, sobre todo con destino a Alemania y Austria, que agotaron el tiempo máximo permitido de siete años ${ }^{11}$. Pero, el aumento de la movilidad pone de manifiesto la existencia de grandes diferencias socioeconómicas entre los lugares de origen y los de acogida, que generan flujos asimétricos de dirección Este-Oeste. Las diferencias tienen raíces en la Guerra Fría y en las dificultades que surgieron durante la transición del sistema comunista al capitalista. En las regiones del Este no hay grandes ciudades cerca de la frontera, salvo excepciones (Bratislava, en Eslovaquia; Frankfurt-Oder, en Polonia), la proporción de población rural es elevada, el índice de desempleo superior a la media estatal y tienen grandes bolsas la pobreza y exclusión social, unas condiciones que empujan a la búsqueda de oportunidades en las regiones más ricas ubicadas en el Oeste (Cavallaro \& Dianin, 2019).

10 El derecho comunitario distingue entre la libre circulación de trabajadores (artículo 39 del Tratado CE), el libre establecimiento de trabajadores no asalariados (artículo 43) y la libre prestación de servicios (artículo 49). Véase la Versión Consolidada del Tratado Constitutivo de la Comunidad Europea. Diario Oficial de las Comunidades Europeas, 24.12.2002. Recuperado a partir de https://www.mapa.gob.es/es/pesca/ayudas-y-subvenciones/instrumento-financiero-de-orientacion-de-la-pesca/17\%20LEGCOM_tcm30-73539.pdf

11 En mayo de 2011, Alemania y Austria eliminaron las restricciones de acceso a los ciudadanos de Polonia, Chequia, Eslovaquia, Hungría, Eslovenia, Estonia, Letonia y Lituania, y en enero de 2014 a los de Rumania y Bulgaria. Los croatas tienen acceso al mercado de trabajo alemán desde 2015, dos años después de que Croacia se incorporara a la UE (julio de 2013); en el caso de Austria a partir de 2020. 
La diferencia de salario en el seno de la UE, con una relación de 1 a 10 tras la incorporación de los nuevos países socios, es el factor más importante. La oportunidad de conseguir mayores ingresos en el exterior es mucho más relevante que los factores de expulsión en origen (Nerb et al., 2009; Wihtol, 2013). Los trabajadores móviles consiguen ingresos más altos de los que obtendrían en el lugar de origen y se ocupan en empleos temporales, inestables, de bajos salarios, donde las posibilidades de progreso son limitadas. Los empleadores consiguen trabajadores dispuestos a ocupar empleos de baja cualificación en la agricultura, la construcción, la hostelería, el servicio doméstico o los transportes, y se muestran satisfechos porque los transfronterizos les proporcionan mano de obra flexible. El aumento de la demanda ha derivado en procesos de reemplazamiento de mano de obra, como se ha observado en el sector agrario alemán, donde los rumanos han sustituido a los polacos, o en el servicio doméstico de Austria, en el que las mujeres checas han sido reemplazadas por trabajadoras de países más pobres, dispuestas a aceptar las condiciones que les imponen (Fries-Tersch et al, 2020; Sert, 2018; Wiesböck, 2016).)

La ausencia de barreras geográficas y unas buenas infraestructuras de transporte facilitan la movilidad. Las conexiones Este-Oeste fueron muy deficientes durante el largo periodo de la Guerra Fría y lo siguen siendo en muchos sectores de la frontera, sobre todo en regiones con débil densidad de población y bajo nivel de desarrollo, que por sí solos frenan los flujos de trabajadores. Las mejoras en las comunicaciones han impulsado la movilidad entre regiones vecinas: Baviera (Alemania) y Bohemia (Chequia), Berlín y Szczecin (Polonia), Trieste (Italia), Liubliana (Eslovenia) y Graz (Austria) y, por supuesto, entre Viena y Bratislava (Eslovaquia). También han aumentado los desplazamientos entre Štúrovo (Eslovaquia) y Esztergom (Hungría) desde la reconstrucción de puentes sobre el Danubio y el Ipel a principios del siglo XXI (Balogh \& Pete, 2017; Cavallaro \& Dianin, 2019; Nerb et al., 2009). La proximidad geográfica es un factor determinante de la movilidad. Así, por ejemplo, los 60 kilómetros que separan Bratislava de Viena se pueden cubrir en menos de una hora, lo que permite viajar a diario entre el lugar de residencia y el de trabajo (Wiesböck, Verwiebe, Reinprecht, \& Haindorfer, 2016; Williams \& Balá, 2002). La corta distancia facilita la vida familiar, sin ruptura de relaciones familiares y sociales en origen. De tal forma que miles de húngaros, polacos, checos o eslovacos se han instalado en la movilidad, trabajan en países de la UE-15, donde consiguen salarios más altos que gastan en el lugar de residencia, donde el coste de la vivienda y el nivel de vida son considerablemente más bajos. En las distancias largas, de centenares e incluso miles de kilómetros, es más habitual la movilidad de corta duración de trabajadores que viajan en avión. Así, por ejemplo, los profesionales de la salud de Europa del Este que trabajan el fin de semana o durante unas semanas o meses en los países escandinavos, el Reino Unido, Bélgica o Francia, viajan en compañías aéreas low cost (Maier et al., 2011). Los trabajadores se desplazan cuando los ingresos obtenidos compensan los costes del desplazamiento.

La proximidad cultural e idiomática facilita la movilidad transfronteriza y la incorporación al mercado de trabajo en el país de acogida, y puede ser un factor decisivo en la elección del destino (Fries-Tersch et al., 2020). Por ejemplo, rumanos en España o Italia, búlgaros en Turquía, checos, eslovacos o húngaros en Austria o estonios en Finlandia (Baumgartner \& Forster, 2017; Drbohlav \& Pavelková, 2018; Sert 2018; Wiesböck, 2016). Cuando no se domina el idioma, los trabajadores 
recurren a las redes sociales para su inserción laboral. En este caso, el capital social compensa la falta de capital humano. La red social es fundamental para comprender la movilidad transfronteriza entre Austria y sus vecinos del Este (Verwiebe et al., 2017). Los compatriotas y familiares ya establecidos en el país de acogida transmiten información al trabajador sobre las oportunidades laborales y actúan de intermediarios entre éste y el empleador, que confía en personas conocidas.

Las empresas de trabajo temporal (ETT) tienen cada vez más importancia en la contratación de trabajadores desplazados. Los empleadores recurren a los servicios de estas empresas para adaptar las plantillas a la demanda y reducir los costes de producción. El margen de beneficios aumenta cuanto más barato es el servicio, por eso el empleador final prefiere las agencias de Europa del Este y de países con sistemas fiscales más ventajosos, como Chipre, Liechtenstein, Irlanda o Luxemburgo. Un gran número de ciudadanos del Este ha trabajado por intermediación de estas empresas en Finlandia, Suecia, Dinamarca, Holanda, Francia, etc. (Danaj \& Sippola, 2015; Maslauskaite, 2014). Muchos trabajadores desconocen el idioma en que está redactado el contrato de trabajo, circunstancia que aprovechan los empleadores para imponer unas condiciones deplorables. Con frecuencia las jornadas se prolongan más de lo habitual, hasta 12 y 14 horas, incluso durante el fin de semana, a cambio de salarios que son considerablemente inferiores a los que obtienen los trabajadores locales (Cremers, 2011). Sin embargo, en muchos casos, el escaso dominio del idioma es el motivo por el que algunos prefieren trabajar con empresas de empleo temporal que, aparte de proporcionarles trabajo, también se ocupan de organizar el desplazamiento y el alojamiento (Ceccagno \& Sacchetto, 2020; Napierała \& Fiałkowska, 2013).

\section{Conclusión}

Los europeos del Este cuentan con una larga experiencia en las migraciones fronterizas y de corta duración, que han aumentado de forma extraordinaria en los últimos años. En el reciente aumento de la movilidad confluyen diversos factores: la globalización económica y la demanda de trabajadores flexibles, las oportunidades económico-laborales en los países de acogida, las diferencias de salario con respecto al país de origen, el papel de las redes sociales, la proximidad geográfica y cultural, y el desarrollo y abaratamiento de los medios de transporte y comunicación. La ampliación de la UE y la libre circulación de trabajadores han dado un gran impulso a los desplazamientos transfronterizos. Probablemente, la movilidad habría registrado niveles más altos sin las restricciones que durante varios años impusieron los países de acogida. Sin embargo, la moratoria ha tenido una influencia limitada en la movilidad Este-Oeste. Los trabajadores han eludido los obstáculos accediendo a los mercados como trabajadores autónomos y trabajadores desplazados. La figura del trabajador desplazado, regulada por la Directiva 96/71/CE, se ha aprovechado para contratar trabajadores temporales que pudieran moverse fácilmente por el territorio europeo sin ningún tipo de restricción, con la ventaja añadida de que proporcionan mano de obra barata. A este respecto, interesa destacar que entre los trabajadores transfronterizos aumenta la presencia ciudadanos no comunitarios. 
La movilidad transfronteriza es la alternativa a la emigración permanente, que implica un elevado coste (económico y emocional) para el migrante y su familia. Es la opción preferida por motivos lingüísticos, culturales, familiares o económicos. Miles de trabajadores se han instalado en la movilidad con el fin de mejorar las condiciones de vida en origen. Las frecuentes idas y venidas entre el lugar de trabajo y el de residencia permiten una doble vinculación espacial, ya que el trabajador forma parte a la vez de la población activa en el país de destino y de la población residente en el de origen.

La libre circulación de personas es una de las libertades fundamentales consagradas en el Tratado de Roma (1958), muy apreciada por los trabajadores de los nuevos países socios, de la que han sacado provecho. Los europeos del Este son los ciudadanos comunitarios más móviles. La movilidad laboral contribuye a equilibrar la oferta y la demanda de trabajadores, es un mecanismo para resolver las ineficiencias de los mercados laborales nacionales. Sin embargo, pese al aumento de los desplazamientos, la movilidad intraeuropea es relativamente escasa. Esto es así porque las personas tienden a buscar trabajo en su propio país, aunque la oferta de empleo exterior se encuentre cerca del lugar de residencia. Esta actitud reduce las oportunidades para los trabajadores y frena la consolidación de un mercado laboral europeo.

Los emigrantes y trabajadores transfronterizos de Europa del Este han contribuido a satisfacer la demanda de mano de obra en Europa occidental, donde constituyen un complemento indispensable de los trabajadores locales (autóctonos e inmigrantes residentes). Sin la participación de los trabajadores itinerantes, la economía de los países de acogida se ve seriamente comprometida, como ha revelado la actual crisis sanitaria (Hooper \& Le Coz, 2020; Kainz, 2020). Las medidas extraordinarias (restricciones de los viajes, cierre de fronteras), adoptadas para frenar la difusión de la Covid-19, han tenido consecuencias en la movilidad geográfica laboral. Pero, los flujos no han desaparecido, como tampoco lo hicieron cuando los países de la UE-15 impusieron moratorias a la libre circulación de los trabajadores de los nuevos socios comunitarios. Pese al cierre de fronteras y la persistencia de la pandemia, los países occidentales han recibido trabajadores del Este -y también de países terceros- en sectores como la agricultura, la sanidad o el cuidado de personas (Andriescu, 2020). La crisis sanitaria ha puesto de relieve la valiosa aportación de los trabajadores fronterizos y desplazados, como reconoce la Comisión Europea en la comunicación de finales de marzo de 2020, en la que recomienda a los países miembros que faciliten la libre circulación ${ }^{12}$. El motivo es evidente: los países receptores no disponen de mano de obra experimentada para reemplazarles. Los recientes contagios en diferentes empresas y regiones con destacada presencia de trabajadores móviles han dado visibilidad a las precarias condiciones de trabajo y alojamiento de este colectivo. Unas condiciones que ya se daban antes de la propagación de la enfermedad.

12 Comunicación de la Comisión: directrices relativas al ejercicio de la libre circulación de trabajadores (2020/C 102 I/03). Diario Oficial de la Unión Europea, 30 de marzo de 2020. Recuperado a partir de https://www.boe.es/ doue/2020/102/Z00012-00014.pdf 


\section{REFERENCIAS}

Andriescu, M. (2020): Under Lockdown Amid COVID-19 Pandemic, Europe Feels the Pinch from Slowed Intra-EU Labor Mobility. Brussels: Migration Policy Institute. Recuperado a partir de https://www. migrationpolicy.org/article/covid19-europe-feels-pinch-slowed-intra-eu-labor-mobility.

Arango, J. (2003): La ampliación de la Unión Europea y las migraciones internacionales. En J. Hay (Comp.), Desafíos. La Unión Europea ante su ampliación (pp. 279-298). Madrid: Siddharth Mehta Ediciones.

Bade, K. J. (2003): Europa en movimiento: las migraciones desde finales del siglo XVIII hasta nuestros días. Madrid: Crítica.

Balogh, P. \& Pete, M. (2018): Bridging the Gap: Cross-border Integration in the Slovak-Hungarian Borderland around Stúrovo-Esztergom. Journal of Borderlands Studies, Vol 33(4), 605-622, https:// doi.org/10.1080/08865655.2017.1294495.

Baumgartner, K. \& Forster, J. (2017): Measuring cross-border working in Austria with the EU-LFS. Copenhagen: 12th Workshop on Labour Force Survey Methodology. Recuperado a partir de https:// www.dst.dk/.../2C-1-Measuring-cross-border-working-in-Austria.

Bioteau, E. \& Michalon, B. (2008): L'élargissement de l'Union européenne à l'Est: Bruxelles au défi des relations transfrontalières. Géoconfluences. Recuperado a partir de http://geoconfluences.ens-lyon. $\mathrm{fr} /$ doc/typespace/frontier/FrontScient 7.htm.

Bogoevski, V. (2016): Posting of workers - strengthening labour rights while securing free movement of services? Multicultural Center Prague. MigrationOnline.cz. Recuperado a partir de https://migrationonline.cz/en/posting-of-workers-strengthening-labour-rights-while-securing-free-movement-services.

Cavallaro, F. \& Dianin, A. (2019): Cross-border commuting in Central Europe: features, trends and policies. Transport Policy, 78, 86-104. https://doi.org/10.1016/j.tranpol.2019.04.008.

Ceccagno, A. \& Sacchetto, D. (2020): The mobility of workers living at work in Europe. Current Soiology, 68(3), 299-315. https://doi.org/10.1177/0011392119863831.

Comisión Europea (2008): Repercusiones de la libre circulación de trabajadores en el contexto de la ampliación de la Unión Europea. COM (2008) 765 final. Recuperado a partir de https://eur-lex.europa.eu/ LexUriServ/LexUriServ.do?uri=COM:2008:0765:FIN:ES:PDF.

Comisión Europea (2020): Directrices relativas al ejercicio de la libre circulación de trabajadores (2020/C 102 I/03). Diario Oficial de la Unión Europea, 30 de marzo de 2020. Recuperado a partir de https:/www.boe.es/doue/2020/102/Z00012-00014.pdf.

Comte, E. (2019): Promising more to give less: international disputes between core and periphery around European posted labor, 1955-2018, Labor History, 60(6), 749-764, https://doi.org/10.1080 10023656X.2019.1642465.

Cremers, J. (2011): À la recherche de main d'oeuvre bon marché en Europe. Conditions de travail et de vie des travailleurs détachés. Bruxelles: European Institute for Construction Labour Research. CLR Studies. Recuperado a partir de http://www.eurodetachement-travail.eu/synthese/doc/JC_CLR6.pdf. 
Cremers, J. (2014): Letter-box companies and abuse of the posting rules: how the primacy of economic freedoms and weak enforcement give rise to social dumping. Brussels: ETUI Policy Brief, No 5/2014. Recuperado a partir de https://www.etui.org/Publications2/Policy-Briefs/.

Cremers, J., Dølvik, J. E. \& Bosch, G. (2007): Posting of workers in the single market: attempts to prevent social dumping and regime competition in the EU. Industrial Relations Journal, 38(6), 524541. https://doi.org/10.1111/j.1468-2338.2007.00462.x.

Dac, M. (2015): La migration polonaise après 1989: entre impératifs économiques et liberté de mouvement retrouvée. Migrations Société, 158, 131-138. Recuperado a partir de https:/www.cairn.info/ revue-migrations-societe-2015-2-page-131.htm.

Danaj, S. \& Sippola, M. (2015): Organizing posted workers in the construction sector. En Jan Drahokoupil (ed.). The outsourcing challenge: organizing workers across fragmented production networks (pp. 217-235). Brussels: ETUI. Recuperado a partir de https://www.etui.org/Publications2/Books/ The-outsourcing-challenge-organizing-workers-across-fragmented-production-networks.

Danaj, S. (2018): Posted Work And Occupational Safety And Health: A Literature Review, Dve Domovini, 48, 37-54. Recuperado a partir de http://twohomelands.zrc-sazu.si/en/issues/articles_ list/48/2018.

De Tinguy, A. (1993): Migrations de l'Est: où en est-on trois ans après la chute du mur de Berlin? Études internationales, 24(1), 141-161, https://doi.org/10.7202/703133ar.

De Wispelaere, F., De Smedt, L. \& Pacolet, J. (2020): Posting of workers. Report on A1 Portable Documents issued in 2018. Brussels: European Commission. Directorate General for Employment, Social Affairs and Inclusion. Recuperado a partir de https://www.mobilelabour.eu/13148/report-on-posting-of-workers-a1-portable-documents-issued-in-2018/.

DIRECTIVA 96/71/CE del Parlamento Europeo y del Consejo, de 16 de diciembre de 1996, sobre el desplazamiento de trabajadores efectuado en el marco de una prestación de servicios. Diario Oficial de las Comunidades Europeas, n L 18, de 21 de enero de 1997. Recuperado a partir de https://eurlex.europa.eu/legal-content/ES/TXT/PDF/?uri=CELEX:31996L0071\&from=ES.

Dølvik, J. E. \& Eldring, L. (2006): Éditorial: La mobilité des services et le détachement des travailleurs dans l'Europe élargie; défis pour la réglementation du marché du travail. Transfer, 2, 142-147, https://doi.org/10.1177/102425890601200202.

Drbohlav, D. \& Pavelková, L. (2018): Intra-European Movement of Czechs with Special Regard to Austria and Care Givers (The "MICO" Type-Between MIgration and COmmuting. En P. Scolten \& M. van Ostaijen (Ed.). Between Mobility and Migration (pp. 205-226). Cham (Switzerland), IMISCOE: Springer International Publishing AG. https://doi.org/10.1007/978-3-319-77991-1_11. EFT (2012): Esclavage moderne dans une Europe moderne? Bruxelles: Fédération Européenne des Travailleurs des Trasnports. Recuperado a partir de https://www.etf-europe.org/wp-content/ uploads/2018/09/ETF-brochure-Modern-slavery-in-modern-Europe-FR.pdf.

EMN (2012): Glosario sobre Migración y Asilo 2.0. Red Europea de Migraciones. Comisión Europea. Recuperado a partir de https://ec.europa.eu/home-affairs/sites/homeaffairs/files/what-we-do/networks/european_migration_network/docs/emn-glossary-es-version.pdf. 
Engbersen, G., Leerkes, A., Grabowska-Lusinska, I., Snel, E. \& Burgers, J. (2013): On the Differential Attachments of Migrants from Central and Eastern Europe: A Typology of Labour Migration. Journal of Ethnic and Migration Studies, 39(6), 959-981. https://doi.org/10.1080/1369183X.2013.765663. European Commission (2008): Scientific Report on the Mobility of Cross-border Workers within the EU-27/ EEA/EFTA countries. Final Report. Annex. Brussels: Employment and Social Affairs.

European Commission (2011): Mobility in Europe. Employment, Social Affairs and Equal Opportunities.

European Commission (2012): Posting of workers in the European Union and EFTA countries: Report on A1 portable documents issued in 2010 and 2011. Brussels: Employment, Social Affairs and Inclusion DG.

EUROSTAT: Employment and commuting by NUTS 2 regions. Recuperado a partir de http://appsso. eurostat.ec.europa.eu/nui/show.do?dataset=lfst_r_lfe2ecomm\&lang=en.

EUROSTAT: Estatistics Explained. Transport Road. Recuperado a partir de https://ec.europa.eu/eurostat/ statistics-explained/index.php?title=Category:Road.

Favell, A. (2008): The new Face of East-West Migration in Europe. Journal of Ethnic Migration Studies 34(5), 701-716. https://doi.org/10.1080/13691830802105947.

Fernandes, S. (2017): Travailleurs détachés: comment garantir une mobilité équitable? Institute Jacques Delors. Recuperado a partir de https://institutdelors.eu/wp-content/uploads/2017/11/travailleursdtachs-tribune-fernandes-oct17.pdf.

Forti, O., Pittau, F. \& Ricci, A. (2004): Europa. Allargamento a Est e immigrazione. Roma: Caritas Italiana.

Fries-Tersch, E., Jones, M., Böök, B., de Keyser, L., \& Tugran, T. (2020): 2019 Annual Report on Intra-EU Labour Mobility. Brussels: European Commission. Directorate General for Employment, Social Affairs and Inclusion. Recuperado a partir de https://ec.europa.eu/social/main.jsp?catI$\mathrm{d}=738$ \& furtherPubs=yes\&langId=en\&pubId=8242.

Hooper, K. \& Le Coz, C. (2020): A Race Against the Clock: Meeting Seasonal Labor Needs in the Age of COVID-19. Brussels: Migration Policy Institute. Recuperado a partir de https://www.migrationpolicy.org/news/meeting-seasonal-labor-needs-age-covid-19.

Kahanec, M., Pytliková, M. \& Zimmermann, K. (2016): The Free Movement of Workers in an Enlarged European Union: Institutional Underpinnings of Economic Adjustment. En M. Kahanec \& K. Zimmermann (Eds.), Labor Migration, EU Enlargement and the Great Recession (pp. 1-34). Berlin-Heildelberg: Springer-Verlag. Recuperado a partir de https://www.iza.org/publications/ $\mathrm{dp} / 8456 /$ the-free-movement-of-workers-in-an-enlarged-european-union-institutional-underpinnings-of-economic-adjustment.

Kainz, L. (2020): As COVID-19 Slows Human Mobility, Can the Global Compact for Migration Meet the Test for a Changed Era? Brussels: Migration Policy Institute. Recuperado a partir de https://www. migrationpolicy.org/news/covid19-global-compact-migration-faces-test. 
Koikkalainen, S. (2011): Free Movement in Europe: Past and Present. Brussels: Migration Policy Institute. Recuperado a partir de https://www.migrationpolicy.org/article/free-movement-europe-past-and-present.

Lends, D., Mussche, N. \& Marx, I. (2019): Europe's ever expanding mobility patterns-posting, third-country nationals and the single European labour market. Working Paper, No. 19.08. Herman Deleeck Centre for Social Policy. University of Antwerp. Recuperado a partir de http://www.centrumvoorsociaalbeleid.be/sites/default/files/CSBWorkingPaper1908.pdf.

Maier, C. B., Glinos, I. A., Wismar, M., Bremner, J. Dussault, G. \& Figueras, J. (2011): Cross-country analysis of health profesional mobility in Europe: the results. En M. Wismar, C. B. Maier, I. A. Glinos, G. Dussault, \& J. Figueras (Eds.), Helath Professional Mobility and Health Systems. Evidence from 17 European countries (pp.23-66). European observatory on Health Systems and Policies, World Health Organization. Recuperado a partir de http://www.euro.who.int/_data/assets/ pdf_file/0017/152324/Health-Professional-Mobility-Health-Systems.pdf?ua=1.

Maslauskaite, K. (2014): Travailleurs détaches dans l'UE: État des lieux et évolution réglementaire. Policy Paper, 107. Institut Jacques Delors. Recuperado a partir de http://www.institutdelors.eu/ wp-content/uploads/2018/01/travailleursdtachs-maslauskaite-ne-ijd-mar14.pdfMorockvasic,.

Mintchev, V., Boshnakov, V., Richter, M. \& Ruspini, P. (2016): Determinants of Migration and Types of Migration and Mobility. En M. Richter, P. Ruspini, D. Mihailov, V. Mintchev \& M. Nollert (Eds.), Migration and transnationalism Between Switzerland and Bulgaria (pp. 25-60). Switzerland: Sringer International Publishing.

Morokvasic, M. (1999): La mobilité trnasnationale comme ressource: le cas des migrants de l'Europe de l'Est. Cultures $\mathcal{E}$ Conflits, 33-34,105-122. https://doi.org/10.4000/conflits.263.

Morokvasic, M. (2015): Migrations et mobilités Est-Ouest après 1989 sur fond d'integration Européenne. Migrations Société, 158, 61-92. https://doi.org/10.3917/migra.158.0061.

Mussche, N., Corluy, V. \& Marx, I. (2016): The Rise of the Free Movements: How Posting Shapes a Hybrid Single European Labour Market. Forschungsinstitut zur Zukunft der Arbeit Institute for the Study of Labor. IZA DP No. 10365. http://ftp.iza.org/dp10365.pdf.

Napierała, J. \& Fiałkowska, K. (2013): Mapping the Market for Employment Agencies in Poland. En Friberg J. Horgen y Eldring, Line (Eds.). Labour Migrants from Central and Eastern Europe in the Nordic Countries (pp. 169-200). Dinamarca: Consejo Nórdico de Ministros. https://oi.org/10.6027/9789289329057-13-en.

Nerb, G., Hitzelsberger, F., Woidich, A., Pommer, S., Hemmer, S. \& Heczko, P. (2009): Scientific Report on the Mobility of Cross-Border Workers Within the EU-27/EEA/EFTA countries. Brussels: European Commission. Recuperado a partir de https:/ec.europa.eu/futurium/en/system/files/ged/mkw_workers_mobility.pdf.

OCDE (2019): Perspectives des Migrations Internationales 2019. Rapport Annuele. Paris: Éditions OCDE. https://doi.org/10.1787/60811ed3-fr.

Okólski, M. (2001a): Últimas tendencias y principales temas de las migraciones internacionales: perspectivas de Europa Central y del Este. Revista Internacional de Ciencias Sociales, 165, 78-90. 
Okólski, M. (2001b): La politique migratoire de la Pologne à la veille de son adhésion à l'Union Européenne. Hommes $\mathcal{G}$ Migrations, 1230, 51-58. Recuperado a partir de https://www.persee.fr/doc/ homig_1142-852x_2001_num_1230_1_3657.

Okólski, M. \& Salt, J. (2014): Polish Emigration to the UK after 2004, Why Did So Many Come? Central and Eastern European Migration Review, 3(2), 11-37. Recuperado a partir de http://www. ceemr.uw.edu.pl/vol-3-no-2-december-2014/articles/polish-emigration-uk-after-2004-why-did-somany-come.

Pajares, M. (2007): Inmigrantes del Este. Procesos migratorios de los rumanos. Barcelona: Icaria.

Perocco, F. (2018): Precarizzazione strutturale del lavoro e precarizzazione globale delle migrazioni. L'esempio dei lavoratori in distacco intracomunitario. Economia e Società Regionale, 36(3), 132-153.

Rea, A. (2013): Les nouvelles figures du travailleur immigré: fragmentation des statuts d'emploi et européanisation des migrations. Revue européenne des migrations internationales, 29(2), 15-35. https:// doi.org/10.4000/remi.6371.

REGLAMENTO (CE) 883/2004 del Parlamento Europeo y del Consejo, de 29 de abril, sobre la coordinación de los Sistemas de Seguridad Social. Diario Oficial de la Unión Europea $n^{\circ}$ L 166, de 30 de abril. Recuperado a partir de https://eur-lex.europa.eu/legal-content/ES/TXT/PDF/?uri=CELEX:0 2004R0883-20140101\& from $=$ ES.

Riesco, A., García, J. \& Maira, M. M. (2018): Desplazamiento de trabajadores en la Unión Europea. El caso del transporte por carretera. Albacete: editorial Bormazo.

Salt, J. (2002): Évolution actuelle des migrations internationales en Europe. Conseil de l'Europe, CDMG. 26. Recuperado a partir de https://www.coe.int/t/dg3/migration/archives/Documentation/Migration\%20management/2002_Salt_report_fr.pdf.

Salt, J. \& Clark, J. (2001): La migración internacional en la región de la CENE: modelos, tendencias y políticas. Revista Internacional de Ciencias Sociales, 165, 64-77.

Sert, D. (2018): The Diversification of Intra-European Movement. En P. Scholten \& M. Van Ostaijen (Eds.). Between Mobility and Migration. The Multi-Level Governance of Intra-European Movement (pp. 21-43). IMISCOE Research Series. Springer Open. Recuperado a partir de https://link.springer.com/content/pdf/10.1007\%2F978-3-319-77991-1.pdf.

Skeldon, R. (2012): "Going Round in Circles: Circular Migration, Poverty Alleviation and Marginality”. International Migration, 50(3), 43-60. https://doi.org/10.1111/j.1468-2435.2012.00751.x.

SOPEMI (1998): Tendances des Migrations Internationales. Rapport Annuele. Paris: Éditions OCDE. Recuperado a partir de https://www.oecd-ilibrary.org/social-issues-migration-health/tendances-des-migrations-internationales-1998_migr_outlook-1998-fr.

Soultanova, R. (2005): Les migrations multiples de la population bulgare. Groupe d'études sur l'etnicité. Bruxelles: Université Libre de Bruxelles. Recuperado a partir de https://www.courrierdesbalkans.fr/ les-migrations-multiples-de-la-population-bulgare.

Sthacher, I. (2001): L'impact de l'élargissement de l'Union Européenne sur l'Autriche. Hommes $\mathcal{E}$ Migrations, 1230, 43-50. Recuperado a partir de https://www.persee.fr/doc/homig_1142-852x_2001_ num_1230_1_3656. 
Stola, D. (1998): Les migrations en Pologne dans les années quatre-vingt-dix. Hommes $\mathcal{E}$ Migrations, 1261, 58-69. Recuperado a partir de https:/www.persee.fr/doc/homig_1142-852x_1998_ num_1216_1_3246.

Trčka, M. (2018): Modern European Guest Workers? Problematic aspects of posting workers and the new Posted Workers Directive. Multicultural Center Prague. MigrationOnline.cz. Recuperado a partir de https://migrationonline.cz/en/e-library/modern-european-guest-workers-problematic-aspects-of-posting-workers-and-the-new-posted-workers-directive.

Verwiebe, R., Reinprecht, C., Haindorfer, R. \& Wiesboeck, L. (2017): How to Succeed in a Transnational Labor Market: Job Search and Wages among Hungarian, Slovak, and Czech Commuters in Austria, International Migration Review, 15(1), 251-286. https://doi.org/10.1111/imre.12193.

Viruela, R. (2004): El recurso de la emigración. Balance durante la transición en Rumania. Papeles del Este, 9. Recuperado a partir de https://dialnet.unirioja.es/servlet/articulo?codigo=1111845.

Wagner, I. (2015): Posted Work and Deterritorialization in the European Union. A study of the German Construction and Meat Industry. University of Jyväskylä: Department of Social Sciences and Philosophy. Recuperado a partir de https://jyx.jyu.fi/handle/123456789/45494.

Weber, M. (1991): Estudios políticos. Madrid: Alianza Editorial.

Wiesböck, L. (2016): A preferred workforce? Employment practices of East-West cross-border labour commuters in the Central European Region, Österreichische Zeitschrift für Soziologie, 41, 391-407, https://doi.org/10.1007/s11614-016-0245-3.

Wiesböck, L., Verwiebe, R., Reinprecht, C. \& Haindorfer, R. (2016): The economici crisis as a driver of cross-border labour mobility? A multi-method perspective on the case of the Central European Region, Journal of Ethnic and Migration Studies, 42(10), 1711-1727. https://oi.org/10.1080/13691 83X.2016.1162354.

Wihtol, C. (2013): El fenómeno migratorio en el siglo XXI. Migrantes, refugiados y relaciones internacionales. México: Fondo de Cultura Económica.

Williams, A. M. \& Balá, V. (2002): Mobilité internationale en Europe centrale, touristes, commerçants et migrants. Revue Européenne des Migrations Internationales, 18(1), 37-65. https://doi.org/10.4000/ remi.1680.

Cómo citar este artículo:

Viruela Martínez, R. (2020). La movilidad geográfica de los trabajadores transfronterizos de Europa del Este en las dos últimas décadas. Cuadernos de Geografía, 105, 7-28.

https://doi.org/10.7203/cguv.105.17477

\section{(c) $(1) \Theta \Theta$}

Este obra está bajo una licencia de Creative Commons Reconocimiento-NoComercial-SinObraDerivada 4.0 Internacional. 